\title{
Stochastic Modelling and Analysis of Sub-pixel Edge Detection*
}

\author{
Kalle Åström, Anders Heyden \\ Dept of Mathematics, Lund University \\ Box 118, S-221 00 Lund, Sweden \\ email: kalle@maths.lth.se andersp@maths.lth.se
}

\begin{abstract}
Stochastic analysis of edge detectors can be made either by theoretical modeling of the image formation process and the edge detectors or by empirical stochastic analysis of the edge locations. In this paper we study and model the image formation process in detail. In particular, the much neglected discretisation process is modelled and taken into account. This makes it possible to define and analyse sub-pixel edge detection. The theoretical results are verified through stochastic analysis of both simulated and real image data.
\end{abstract}

\section{Introduction}

Edge detection algorithms have been studied by many researchers in computer vision. One frequently used algorithm which is simple to understand is the Hildreth and Marr edge detector, see [8]. Another popular one is the CannyDeriche edge detector based on an optimality condition, suggested in [3] and effectively implemented in [7]. See also [11] and [12] for an overview ot different edge detectors. The one we will use for our study is similar to [8].

One drawback of these edge detectors is that their statistical properties in the presence of noise have not been properly studied. Canny has calculated the variance of the position of the detected edge in the one-dimensional case, see [3], but has not taken into account that the images are discrete and how this discrete representation of an edge is obtained from the real world. Another attempt can be found in [6], where a comparison of different corner detectors are made. However the analysis is mostly done from simulations and lacks a thorough analysis of the statistics involved.

Our aim is to derive the statistical properties of a simple edge detector. This means to give not only the estimated location of the edge but also some accuracy measure. We will use an edge detector based on the maximal gradient perpendicular to the edge. However the actual edge detector

\footnotetext{
*This work has been supported by the Swedish Research Council for Engineering Sciences (TFR), project 95-64-222
}

used is not as important as the techniques used to analyse it, which can be carried through for other detectors as well.

\section{Image acquisition}

The following image acquisition model will be used,

$$
W_{\text {ideal }} \stackrel{\text { blur }}{\longrightarrow} \stackrel{\text { sampling }}{\longrightarrow} w_{0} \stackrel{\text { noise }}{\longrightarrow} v_{0}
$$

cf. [2], where upper case letters, $W$, denote signals with continuous parameters, whereas lower case letters, $w$, denote discrete signals. Here, and often in the sequel, we use the word signal synonymously with function, and discrete signal synonymously with sequence or function defined on $\mathbb{Z}^{n}$, for some $n$. These three steps of blurring, sampling and addition of noise will now be discussed in a little more detail.

Here blurring is modelled as an abstract operator $h$, such that $W=h\left(W_{\text {ideal }}\right)$. We assume that no aliasing effects are present, when the function $W$ is sampled at integer positions, i.e. $W \in \mathcal{B}\left(\mathbb{R}^{n}\right)$, where

$$
\mathcal{B}\left(\mathbb{R}^{n}\right)=\left\{W \in L_{2}\left(\mathbb{R}^{n}\right) \mid \operatorname{supp} \mathcal{F} W \subset(-1 / 2,1 / 2)^{n}\right\} .
$$

In the definition of the Fourier transform, we use the formula

$$
\mathcal{F} W(f)=\int_{\mathbb{R}^{n}} W(\tau) e^{-i 2 \pi f \cdot \tau} d \tau
$$

where $f \cdot \tau$ denotes scalar product.

The sampling is assumed to be ideal. Introduce the sampling or discretisation operator, $D: \mathcal{B} \rightarrow l_{2}$,

$$
w(i, j)=(D W)(i, j)=W(i, j) .
$$

Note that the sampling operator maps a continuous signal $W$ onto a discrete signal $w$.

Finally noise is assumed to be an additive stationary random field. Experimentally, it is verified that the errors in individual pixel intensities often can be modelled as independent random variables with similar distribution. 


\section{Interpolation and smoothing}

The main idea of our approach is to induce the discrete signal, the scale spaces, etc. from the associated interpolated quantities. By an interpolation or restoration method we mean an operator that maps a discrete signal, $w$, to a continuous one, $W$. The following types of interpolation operators $I_{F}$ will be used:

$$
W(s)=\left(I_{F} w\right)(s)=\sum_{i} F(s-i) w(i) .
$$

We propose to use ideal low-pass interpolation

$$
I=I_{\text {sinc }}
$$

and discretisation $D$ as mappings between the continuous and discrete signals to solve the restoration and discrete scale-space problems. In other words we relate the discrete and continuous signals through the operations of discretisation and ideal low-pass interpolation. Note that if the camera induced blur cancels the high frequency components in $W$ as in (2), the deterministic restoration $W_{0}=I\left(w_{0}\right)$ is equal to $W$.

Introduce the smoothing operator $S_{b}$ representing convolution with the Gaussian kernel

$$
G_{b}(x)=C_{n} e^{-|x|^{2} / 2 b^{2}}
$$

where $C_{n}$ is a constant chosen so that the $L_{2}$ norm is one. Using these definitions, the discrete and continuous scalespace representations can be defined simultaneously and consistently, as illustrated by the diagram:

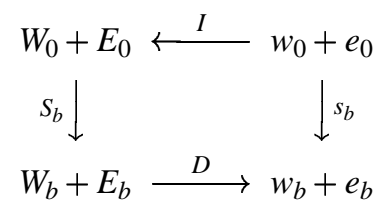

Note that all operations are linear. The stochastic and deterministic properties can therefore be studied separately and the final result is obtained by superposition. Thus with an a priori model on $W_{\text {ideal }}$, for example an ideal edge or corner, it is possible to predict the deterministic parts $W_{b}$ and $w_{b}$. The stochastic properties of the error fields $e_{0}, e_{b}, E_{0}$ and $E_{b}$, will now be studied. The theory of random fields is a simple and powerful way to model noise in signals and images. Stationary random fields are particularly easy to use. Denote by $\mathcal{E}$ the expectation value of a random variable.

Definition 3.1. A random field $X(t)$ with $t \in \mathbb{R}^{n}$ is called stationary, if its mean $m(t)=m_{X}(t)=\mathcal{E}[X(t)]$ is constant and if its covariance function $r_{X}\left(t_{1}, t_{2}\right)=\mathcal{E}\left[\left(X\left(t_{1}\right)-\right.\right.$ $\left.\left.m\left(t_{1}\right)\right)\left(X\left(t_{2}\right)-m\left(t_{2}\right)\right)\right]$ only depends on the the difference $\tau=t_{1}-t_{2}$.
For stationary fields we will use $r_{X}(s, t)$ and $r_{X}(s-t)$ interchangeably as the covariance function. The analogous definition is used for a stationary field in discrete parameters. The notion of spectral density

$$
R_{X}(f)=\left(\mathcal{F} r_{X}\right)(f)=\int r_{X}(\tau) e^{-i 2 \pi f \cdot \tau} d \tau
$$

is also important. Again the same definition can be used for random fields with discrete parameters $s \in \mathbb{Z}^{n}$, but whereas the spectral density for random fields with continuous parameters is defined for all frequencies, $f$, the spectral density of discrete random fields is only defined on an interval $f \in[-1 / 2,1 / 2]^{n}$. Introductions to the theory of random processes and random fields are given in $[1,4,5]$. In these books you will find that convolution, discretisation and derivation preserves stationarity. The effect of these operations on the covariance function is also known, see [2]:

$$
\begin{array}{ccc}
w=D(W) & \Rightarrow & r_{w}=D\left(r_{W}\right) \\
Y=h * X & \Rightarrow & R_{Y}=R_{X}|\mathcal{F} h|^{2} \\
Y=X^{\prime} & \Rightarrow & r_{Y}=-r_{X}^{\prime \prime} \\
W=I(w) & \Rightarrow & r_{W}=I\left(r_{w}\right)
\end{array}
$$

\section{Edge detection}

To analyse sub-pixel edge detectors we model the edge $W_{\text {ideal }}$ and the blur operator $h$. For simplicity, the edge is modelled as an ideal step function, i.e. as the Heaviside function with height $A$ and the smoothing operator $h$ is modelled as convolution with kernel $h$, which is assumed to fulfill (2), and to be approximately a Gaussian of width $a$,

$$
h_{a} \approx G_{a}
$$

The discrete image is then analysed through ideal interpolation and smoothing: $V_{b}=S_{b}\left(I\left(v_{0}\right)\right)$. It is quite popular to define edges as points where the gradient magnitude is maximal in the direction of the gradient, i.e.

$$
\left(\nabla V_{b}\right)^{T}\left(\nabla^{2} V_{b}\right) \nabla V_{b}=0
$$

cf. [9]. Several simplifications will be made. We will study the stability of edges with respect to a given search direction $\tilde{n}$, i.e. edges are defined as points where

$$
(\tilde{n})^{T}\left(\nabla^{2} V_{b}\right) \tilde{n}=0 \text {. }
$$

Let the true edge $\gamma$ be parametrised by curve parameter $\tau$. Apply the edge detector in search direction $\tilde{n}$ from every point $\gamma(\tau)$. The detected edge can then be parametrised as $\tilde{\gamma}(\tau)=\gamma(\tau)+z(\tau) \tilde{n}$, where $z$ describes the deviation of the detected edge from the true edge.

An ideal edge is modelled as

$$
W_{\text {ideal }}(x, y)=A \theta(x)
$$


where $\theta$ is the Heaviside function. The deterministic discretised image $w_{0}$ of this edge is

$$
w_{0}(i, j)=D\left(h * W_{\text {ideal }}\right)(i, j) \approx A \Phi_{a}(i)
$$

where $\Phi_{a}$ is the one-dimensional normal cumulative distribution function. Below the scale-space interpolation $W_{b}=$ $S_{b}\left(I\left(w_{0}\right)\right)$ will be studied.

$$
W_{b}=S_{b}\left(h * W_{\text {ideal }}\right) \approx S_{c} W_{\text {ideal }},
$$

where $c=\sqrt{a^{2}+b^{2}}$. In the two-dimensional case we estimate the edge as the locus of the points where the directional derivative has a local maxima on a line with direction $\tilde{\mathbf{n}}$. If we approximate $W_{b}$ as above we find that it is constant along the edge. Consider the derivatives in the direction $\tilde{\mathbf{n}}=(\cos (\alpha), \sin (\alpha))$, where $\alpha$ denotes the angular difference between the search direction $\tilde{\mathbf{n}}$ and the normal $\mathbf{n}=$ $(1,0)$ to the edge. Introduce

$$
F(s)=W_{b}(s \cos (\alpha), s \sin (\alpha)) .
$$

In the following analysis we will need $F$ and its first three derivatives:

$$
\begin{aligned}
F(s) & =\left(W_{\text {ideal }} * G_{c}\right)(s \cos (\alpha)), \\
F^{\prime}(s) & =A \cos (\alpha) G_{c}(s \cos (\alpha)), \\
F^{\prime \prime}(s) & =A \cos ^{2}(\alpha)\left(-\frac{s \cos (\alpha)}{c^{2}}\right) G_{c}(s \cos (\alpha)), \\
F^{\prime \prime \prime}(s) & =A \cos ^{3}(\alpha)\left(-\frac{1}{c^{2}}+\frac{(s \cos (\alpha))^{2}}{c^{4}}\right) G_{c}(s \cos (\alpha)) .
\end{aligned}
$$

Notice that the first derivative has a maximum for $t=0$, independently of $\alpha$, since $F^{\prime \prime}(0)=0$ and the slope of the second order derivative at the zero crossing is

$$
F^{\prime \prime \prime}(0)=-\frac{A \cos ^{3}(\alpha)}{c^{3} \sqrt{2 \pi}}<0
$$

\section{Random part}

Assume that discrete white noise $e_{0}$ is added to the image. According to (12) and (11), the scale-space interpolated error field $E_{b}=G_{b} * I\left(e_{0}\right)$ then is stationary with covariance

$$
r_{E_{b}}(\tau)=\operatorname{sinc} * G_{b \sqrt{2}} .
$$

For large $b$ this can be approximated by

$$
\operatorname{sinc} * G_{b \sqrt{2}} \approx \varepsilon^{2} \frac{1}{4 \pi b^{2}} e^{-|\tau|^{2} / 4 b^{2}} .
$$

The covariance function is the covariance between the intensity at two positions $(x, y)$ and $\left(x+\tau_{x}, y+\tau_{y}\right)$. We also need the covariance functions of the first three directional derivatives of $E_{b}(s, t)$. Since this random field is approximately isotropic for large $b$, it is sufficient to calculate the directional derivatives in the $s$-direction. It then follows from (12) that

$$
r_{E_{s s}^{\prime \prime}}(\rho)=\frac{\partial^{4} r_{E}}{\partial^{4} s}(s, t)
$$

Calculating the derivatives gives

$$
r_{E_{s s}^{\prime \prime}}(s, t)=\frac{\varepsilon^{2} e^{-\left(s^{2}+t^{2}\right) / 4 b^{2}}}{\pi}\left(\frac{3}{16 b^{6}}-\frac{3 s^{2}}{16 b^{8}}+\frac{s^{4}}{64 b^{10}}\right),
$$

The variance is given by

$$
r_{E_{s s}^{\prime \prime}}(0,0)=\frac{3 \varepsilon^{2}}{16 b^{6} \pi}
$$

The random fields $E_{s}^{\prime}, E_{s s}^{\prime \prime}$ and $E_{s s s}^{\prime \prime \prime}$ have zero mean. Furthermore, $E_{s s}^{\prime \prime}(0,0)$ and $E_{s s s}^{\prime \prime \prime}(0,0)$ are independent, cf. [1]. The analysis above is only valid when we search in a direction perpendicular to the edge. If the search direction forms the angle $\alpha$ with the edge normal, we have to evaluate $r_{E_{s s}^{\prime \prime}}(s, t)$ and the other directional derivatives in $(s, t)=$ $(\tau \cos (\alpha), \tau \sin (\alpha))$, just as in the deterministic case. This gives the following expressions for the dependencies of the directional derivatives along the line

$$
r_{E_{s s}^{\prime \prime}}(\tau)=\frac{\varepsilon^{2} e^{-\tau^{2} / 4 b^{2}}}{\pi}\left(\frac{3}{16 b^{6}}-\frac{3 \tau^{2} \sin ^{2} \alpha}{16 b^{8}}+\frac{\tau^{4} \sin ^{4} \alpha}{64 b^{10}}\right),
$$

\section{Analysis of two-dimensional edges}

The distribution of the edge location can now be calculated. The second order directional derivative, $F^{\prime \prime}(x)$, also depends on the angle $\alpha$ between the search direction and the edge normal. Close to the edge position, $F^{\prime \prime}(x)$ can be approximated by the line

$$
y=k x=F^{\prime \prime \prime}(0) x=-\frac{A \cos ^{3}(\alpha)}{c^{3} \sqrt{2 \pi}} x .
$$

The second order derivative calculated from the noisy image is again approximated near the zero crossing by a line

$$
V_{b}^{\prime \prime}(x)=K x+M,
$$

where

$$
\left\{\begin{array}{l}
K=V_{b}^{\prime \prime \prime}(0,0) \\
M=V_{b}^{\prime \prime}(0,0)
\end{array}\right.
$$




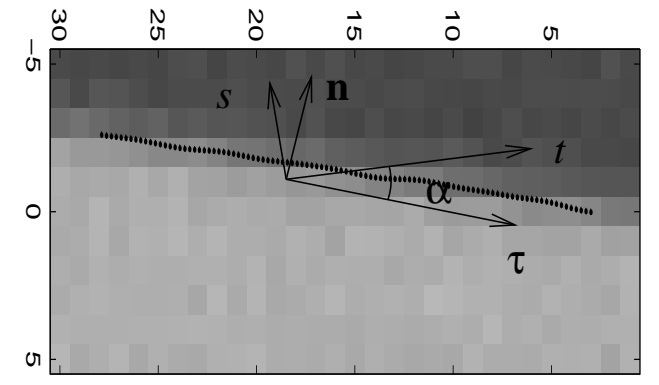

Figure 1. The figure illustrates some notations used in the analysis of two-dimensional edge detection.

are random variables with

$$
\left\{\begin{array}{l}
\mathcal{E}[K]=W_{b}^{\prime \prime \prime}(0)=-\frac{A \cos ^{3}(\alpha)}{c^{3} \sqrt{2 \pi}}, \\
\mathcal{E}[M]=W_{b}^{\prime \prime}(0)=0, \\
\mathcal{V}[M]=r_{E_{s s}^{\prime \prime}}(0,0)=\varepsilon^{2} \frac{3}{16 b^{6} \pi} .
\end{array}\right.
$$

This line (26) has the zero-crossing

$$
X=-\frac{M}{K},
$$

which is a random variable. The probability distribution of $X$ can be approximated by the normal distribution $N(m, \sigma)$ with

$$
m=-\frac{\mathcal{E}[M]}{\mathcal{E}[K]}=0, \quad \sigma=\frac{\mathcal{V}[M]}{\mathcal{E}[K]^{2}},
$$

where Gauss approximation formulas are used together with the fact that $M$ and $K$ are independent, and $\mathcal{E}[M]=0$. Combining (28) and (29) gives

$$
\mathcal{V}[X] \approx \mathcal{V}[M] / \mathcal{E}[K]^{2}=\varepsilon^{2} \frac{3\left(a^{2}+b^{2}\right)^{3}}{8 A^{2} b^{6} \cos ^{6}(\alpha)} .
$$

This is the estimated variance of the detected edge. Observe that the variance decreases with increasing height, $A$, of the edge. The variance also increases when $\alpha$ increases, that is when we do not search perpendicularly to the line.

\section{The detected edge as a random process}

Let the true edge $\gamma$ be parametrised by curve parameter $\tau$. Apply the edge detector in search direction $\tilde{n}$ from every point $\gamma(\tau)$. The detected edge can then be parametrised as $\tilde{\gamma}(\tau)=\gamma(\tau)+z(\tau) \tilde{n}$, where $z$ describes the deviation of the

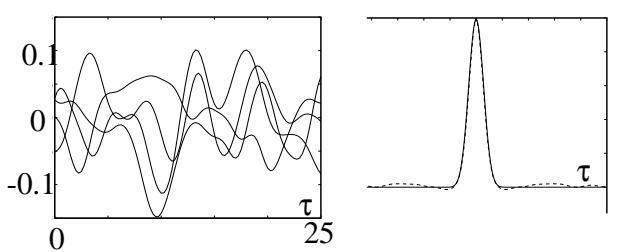

Figure 2. Results from edge detection with simulated data. Left: Edge position errors $z_{i}(\tau)$ at different positions $\tau$ along the edge for four simulations. Right: Theoretical and estimated covariance functions for the residual error process.

detected edge from the true edge. The deviation $z(\tau)$ can be approximated as

$$
z(\tau)=\frac{\left(V_{b}\right)_{s s}^{\prime \prime}(\gamma(\tau))}{\left(W_{b}\right)_{s s s}^{\prime \prime \prime}(\gamma(\tau))}
$$

where

$$
\left(W_{b}\right)_{s s s}^{\prime \prime \prime}(\gamma(\tau))=-\frac{A \cos ^{3}(\alpha)}{c^{3} \sqrt{2 \pi}} .
$$

Then the covariance between the deviations $z\left(\tau_{1}\right)$ and $z\left(\tau_{2}\right)$ is

$$
\begin{aligned}
& C\left[z\left(\tau_{1}\right), z\left(\tau_{1}\right)\right]= \\
& \quad C\left[\left(V_{b}\right)_{s s}^{\prime \prime}\left(\gamma\left(\tau_{1}\right)\right),\left(V_{b}\right)_{s s}^{\prime \prime}\left(\gamma\left(\tau_{2}\right)\right)\right] \frac{c^{6} 2 \pi}{A^{2} \cos ^{6} \alpha},
\end{aligned}
$$

where

$$
\begin{aligned}
c\left[\left(V_{b}\right)_{s s}^{\prime \prime}\left(\gamma\left(\tau_{1}\right)\right),\left(V_{b}\right)_{s s}^{\prime \prime}\left(\gamma\left(\tau_{2}\right)\right)\right]= \\
r_{E_{s s s}^{\prime \prime \prime}}\left(\left(\tau_{1}-\tau_{2}\right) \sin \alpha .\left(\tau_{1}-\tau_{2}\right) \cos \alpha\right)
\end{aligned}
$$

Hence, $z$ is a stationary process with covariance function

$$
\begin{aligned}
& r_{z}(\tau)=r_{E_{s s}^{\prime \prime}}(\tau \sin (\alpha), \tau \cos (\alpha)) / F^{\prime \prime \prime}(0)= \\
& =\frac{2 \varepsilon^{2} c^{6} e^{-\frac{\tau^{2}}{\left(4^{2}\right)}}}{A^{2} \cos ^{6} \alpha}\left(\frac{3}{16 b^{6}}-\frac{3(\tau \sin \alpha)^{2}}{16 b^{8}}+\frac{(\tau \sin \alpha)^{4}}{64 b^{10}}\right) .
\end{aligned}
$$

Notice that the parameter $\tau$ is measured as the arclength along the line. Since the edge is detected as the solution to the equation $W_{b}^{\prime \prime}=0$, we can regard the edge as a level set to $W^{\prime \prime}$. This makes it possible to use a more refined analysis than the approximation with the tangent line described above. This is discussed in detail in [10]. 


\section{Implementation and experiments}

The two-dimensional edge detector described above has been implemented. Its performance on both simulated and real images have been investigated. In the simulations the true edge was well defined. The deviations $z$ were studied both with respect to different realisations but also as a random process along the edge.

The discrete image was disturbed with simulated Gaussian uncorrelated noise. This image was then used in the edge detection routines to calculate the edges along lines roughly perpendicular to the true edge. In these simulations the search line was 0.022 radians off the normal. This was done at several positions along the true edge. The theoretical and empirical standard deviations agree well. Figure 2 illustrates four different realisations of the deviation $z$ in search direction, from the true edge as we move along the edge. The deviation $z(\tau)$ is a stationary random processes. Edge position errors at distance $\tau$ have covariance $r_{z}(\tau)$. The theoretical and empirical covariance functions agree well, as can be seen in Figure 2.

This has also been verified with real data, see Figure 3. In the experiment with real data, five images were taken of the same planar curve. The edge curve was then extracted from each of the five images and aligned. The residuals $z_{i}$ in the normal direction with respect to arclength $\tau$ is shown in Figure 3.

Our analysis was based on ideal, straight step discontinuities. In this case the edge detector is unbiased. Bias is expected for more realistic situations and at edge points with considerable curvature.

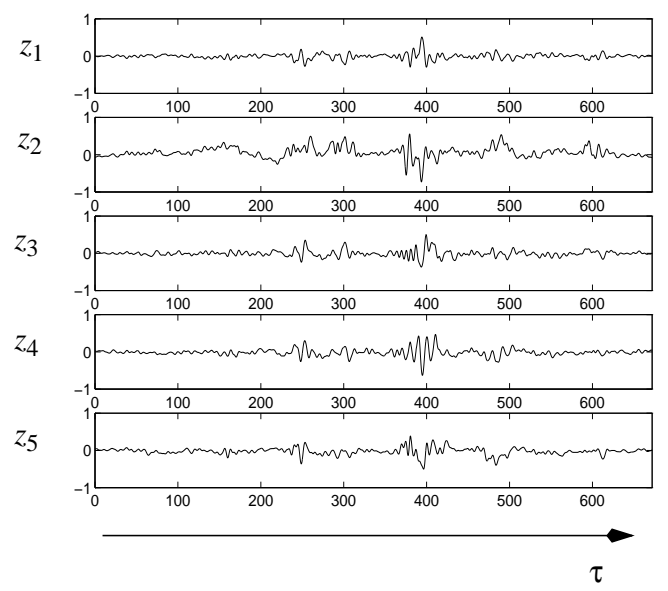

Figure 3. Five edge residuals $z_{i}(\tau)$, empirically estimated after projective alignment of the extracted contours from five different images of the same curve.

\section{Conclusions}

In this paper we have analysed the accuracy of a simple sub-pixel edge detector. We have shown that the location of the edge at different positions along the edge can be regarded as a random process. Furthermore the covariance function of this random process can be calculated and expressed in the variance of the noise, the widths of the Gaussian kernels and the search angle relative to the true normal of the line. In order to validate the theory, experiments and simulations both on real and simulated data have been presented. High agreement with the theoretical model is achieved.

The work can be extended in several directions. In this paper edges were modelled as straight ideal step edges. It would be interesting to study the effect (the bias) caused by other types of edges and the effect of high curvature edges. The model of image acquisition, interpolation and scale space smoothing can also be used to analyse other feature detectors.

\section{Acknowledgement}

The authors thank Georg Lindgren who helped us get going and taught us the basics of random fields.

\section{References}

[1] A. Adler. The Geometry of Random Fields. Wiley, New York, 1985.

[2] K. Åström and A. Heyden. Stochastic analysis of scale-space smoothing. In Proc. International Conference on Pattern Recognition, Vienna, Austria, 1996.

[3] F. Canny. A computational approach to edge detection. IEEE Trans. Pattern Analysis and Machine Intelligence, 8(6):676698, 1986.

[4] H. Cramér and M. R. Leadbetter. Stationary and Related Stochastic Processes. Wiley, New York, 1967.

[5] N. A. C. Cressie. Statistics for Spatial Data. Wiley, New York, 1991.

[6] E. De Michelli, B. Caprile, P. Ottonello, and V. Torre. Localization and noise in edge detection. IEEE Trans. Pattern Analysis and Machine Intelligence, 10:1106-1117, 1989.

[7] R. Deriche. Using Canny's criteria to derive an optimal edge detector recursively implemented. Int. Journal of Computer Vision, 1:167-187, 1987.

[8] E. Hildreth and D. Marr. Theory of edge detection. Proceedings of Royal Society of London, 207:187-217, 1980.

[9] T. Lindeberg. Scale-Space Theory in Computer Vision. Kluwer Academic Publishers, 1994.

[10] G. Lindgren and I. Rychlik. How reliable are contour curves - confidence sets for level contours. Bernoulli, 1(2), 1995.

[11] V. S. Nalwa and T. O. Binford. On detecting edges. IEEE Trans. Pattern Analysis and Machine Intelligence, 8:699714, 1986.

[12] V. Torre and A. Poggio. On edge detection. IEEE Trans. Pattern Analysis and Machine Intelligence, 8:147-163, 1986. 